\title{
Left Ventricular Myxoma: Case Report
}

Holanda Filho Luiz ${ }^{1}$, Arruda Filho Mauro ${ }^{2}$, Martins Isabela ${ }^{3}$, Gomes Hugo ${ }^{4}$, Siqueira Mariana ${ }^{4}$ and Brito Evandro 5,6

1.Instituto de Medicina Integral Professor Fernando Figueira (IMIP), Recife 50070550, Brazil

2.Pronto-Socorro Cardiológico Universitário de Pernambuco (PROCAPE), Universidade de Pernambuco, Recife 50100060, Brazil

3.Scientific, Centro Educacional Maurício de Nassau, Recife 51021140, Brazil

4.General medicine resident, Santa Casa de Misericórdia do Recife, Recife 50040000, Brazil

5.Cardiology, Hospital Agamenon Magalhães, Recife 52070230, Brazil

6. General medicine, Instituto de Medicina Integral Professor Fernando Figueira (IMIP), Recife 50070550, Brazil.

\begin{abstract}
Male, 22 years old, hospitalized in a reference hospital, was diagnosed with a left ventricular myxoma, and underwent into surgical removal of the tumor. The left ventricular myxoma is a rare condition where very few hospitals in the world have experienced in its resolution, making the advancement on its management studies difficult. This article's goal is to promote an update about this subject.
\end{abstract}

Key words: Myxoma, left ventricular, surgical.

\section{Introduction}

Cardiac tumors are very rare in general, being divided into primary and secondary tumors, they are present varied clinical manifestations depending on their topography and cardiac impairment degree.

Up to 2014, at least 71 other surgical cases of left ventricular myxoma (excluding myxomas of the mitral or aortic valve) have been reported, most of them as individual cases. The great difficulty of the management of the left ventricular myxoma is the little experience in the surgical procedure becoming an important challenge

\section{Case Report}

Male, 22 years old, admitted in a reference hospital in the state of Pernambuco (PE), Brazil, presents a history of progressive dyspnea. At the moment of the admission he had dyspnea on minor efforts. On physical examination of the cardiovascular system, he presented regular cardiac rhythm, increased

Corresponding author: Luiz Holanda Filho, scientific, Instituto de Medicina Integral Professor Fernando Figueira (IMIP), research fields: cardiology. cardiovascular fremitus and absent murmur with no edema on the lower extremities.

A transthoracic echocardiogram (TTE) (Fig. 1) was performed, which revealed an intracardiac mass in the left ventricle (LV) adhered to the endocardium in the lateral region (apical segment) of irregular outline, “gelatinous" texture, measuring around $4.5 \times 1.7 \mathrm{~cm}$ in diameter, with an area of approximately $7.27 \mathrm{~cm}^{2}$, projecting at systole to the left ventricular outflow tract (LVOT) without causing significant obstruction to blood flow. Exeresis of the mass was indicated.

For the excision of the tumor a left atriotomy is performed, through the mitral valve (Fig. 2), having been identified as a sessile tumor however with high insertion, demanding a discreet left ventriculotomy in order to excise the tumor.

Macroscopically, the tumor measured approximately $5.0 \mathrm{~cm}$ in its greatest diameter. In order to remove any possible fragments left during the extraction of the tumor an intracavitary lavage was performed, followed by a ventriculography with bovine pericardial patch and left atriorraphy. The material was sent to histopathological analysis, which 
identified irregular, elastic, yellow-brown tissue formations, whose greater formation measured $1.5 \times$ $1.0 \times 0.7 \mathrm{~cm}$ (Fig. 3).

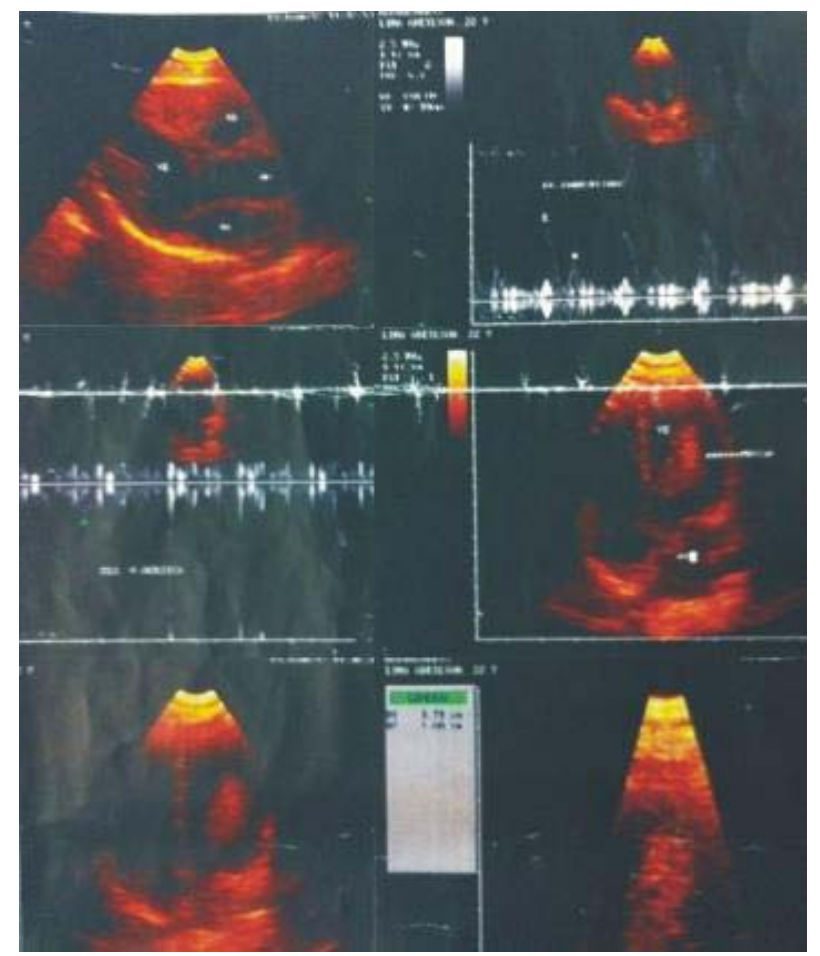

Fig. 1 Transthoracic echocardiogram.

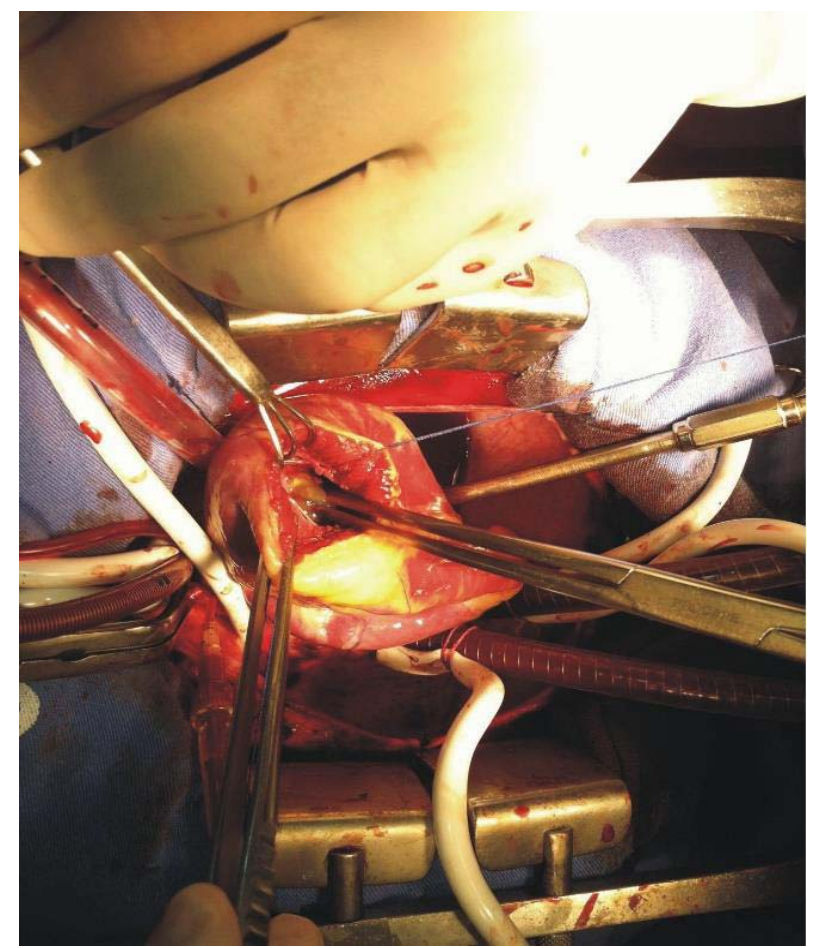

Fig. 2 Picture of the excision of the tumor during cardiac surgery.

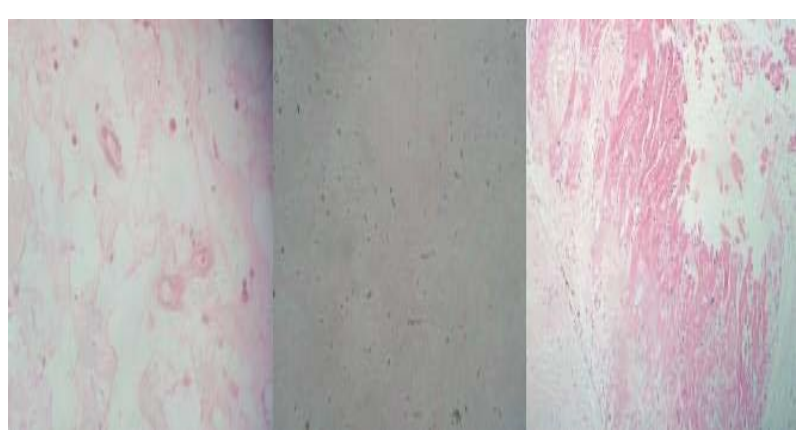

Fig. 3 Histopathological image.

The patient progressed in the postoperative period with recent memory lapses, being examined by the neurology team, which came to the diagnostic hypothesis of transoperative hippocampal hypoxia. He was discharged after a month of hospitalization, clinically well and with no complaints.

\section{Discussion}

Cardiac tumors are very rare in general, being divided into primary and secondary tumors.

The primary type is infrequent [1], having its incidence in general population of $0.0017 \%$ [2] as have been demonstrated by necropsy studies in United States of America. On the other hand, secondary tumors, also called metastatic tumors, are a hundred times more frequent than the primary ones [1].

Myxomas arise from the endocardium and it is generally believed that they are true neoplasms derived from embryonal rests [3]. They are the most frequent cardiac tumors, happening around 40\%-70\% of all cases [1], appearing usually at adulthood and being rare in children $[4,5]$. More than $80 \%$ of myxomas emerge in the left atrium, the majority of them being solitary [1], $15 \%$ to $20 \%$ appear on the right atrium and only $4 \%$ to $2.5 \%$ occur on the left and right ventricle [6]. Myxomas usually have solid or papillary pattern even when located in an atypical position. Multilocular myxomas are rare and usually seen in recurrence [4].

The first documentation of a surgical case of a left ventricle myxoma excision was made by Kay et al. [7] in 1959. By 2014 at least 71 surgical cases of left 
myxoma were reported (excluding mitral or aortic valve myxomas), the majority of them being individual cases [7].

Both primary and secondary tumors present varied clinical manifestations depending on their topography and cardiac impairment degree. The diagnosis can be made incidentally through imaging studies in asymptomatic patients. Many of the symptoms are nonspecific and may mimic different systemic conditions. The findings on physical examination can be similar to those on mytral or tricuspid valve diseases or even present signs of heart failure. The most common symptom is dyspnea, followed by palpitations and weight loss.

Alterations in chest X-rays and electrocardiograms are not frequent, requiring evaluation using echocardiogram which is indispensable to the diagnosis of intracardiac masses. Such method easily and noninvasively provides accurate, real-time information on the size, location and mobility of cardiac masses [8]. If necessary, the investigation can be complemented by computed tomography (CT) or cardiac magnetic resonance imaging (MRI) $[9,10]$.

Treatment of cardiac benign tumors is surgical in most cases [1]. Surgical excision of myxomas is generally considered curative. Atypical primary sites, incomplete excision, metastasis, multicentricity and Carney complex (a hereditary syndrome characterized by multiple myxomas) are known risk factors for recurrency after surgery $[11,12]$.

\subsection{Left Atrium Myxoma}

Atrial myxomas are detectable in $0.3 \%$ of all autopsies and women are affected three times more than men [7]. Cardiac myxomas are more common in adults, representing $80 \%$ of all primary heart tumors [2].

Left atrium myxomas characteristically present the following symptoms: dizziness, palpitations, dyspnea, fever, fatigue and occasionally weight loss $[8,13]$. Systemic embolization is the most common complication of myxomas. More than two thirds of the myxedema embolism cases may migrate to the central nervous system, causing neurological manifestations, including strokes, hemorrhages, aneurysms and metastases $[14,15]$.

The findings on the echocardiogram help not only the detection of the tumor, but the hemodynamic evaluation, mobility, consistency and to assess the base of the tumor as well $[2,16]$.

\subsection{Right Atrium Мухота}

Cardiac myxomas afflict the right atrium in $20 \%$ of the cases, with a ratio of $4: 1$ when compared to the left atrium myxoma. Epidemiologically, cardiac myxomas are more common in the occasional form $(90 \%)$ than the familiar one $(10 \%)[8,14,17]$.

Morphologically, atrial myxomas are characterized by being sessile. Depending on the size and location, a tricuspid prolapse may occur. The clinical presentation of myxomas in right chambers is marked by hemodynamic imbalance due to right intracardiac obstruction as well as signs and symptoms of pulmonary embolism and general systemic symptoms similar to those observed in the left chamber. Moreover, in rare occasions, it is possible to observe systemic embolism symptoms, such as the case of the systemic paradoxical embolism with tumoral lesion in the right atrium which reaches the main circulation as a result of a coexistent interatrial communication $[8,9$, $18]$.

\subsection{Right Ventricle Myxoma}

The right ventricular myxoma has low tumor prevalence, and when they occur, they are usually sarcomas, and myxomas are only described in rare cases (5\%) [9].

In the epidemiological context, it is noted that the ventricular arrangement of myxomas is more characteristic in young women $[6,8,17]$. Morphologically, there are tumors that are mostly pediculated and depending on their size and location, 
pulmonary prolapse may occur. This condition may lead to syncope or sudden death due to obstruction of the outflow. The symptomatology when there is no hemodynamic complication resembles those of the classical triad $[8,9,19]$.

\subsection{Left Ventricle Myxoma}

Up to 2014, at least 71 other surgical cases of left ventricular myxoma (excluding myxomas of the mitral or aortic valve) have been reported, most of them as individual cases [7].

The clinical symptoms of left ventricular myxoma are more commonly presented as syncope and dyspnea and depend on the following mechanisms: interference in the functioning of the mitral and aortic valves; invasive tumors that can cause arrhythmias, pericardial effusion or even cardiac tamponade; systemic embolism is seen in $50 \%$ of cases [4, 20]. Acute occlusion of the aorta can occur, being rare in the literature, and may present as paraplegia (due to spinal cord ischemia) in $50 \%$ of the cases and is incorrectly diagnosed as a neurological disorder [3]. In the case of the patient dyspnea in minor efforts was the main factor to go to the hospital, showing the importance of evaluation of the cardiovascular system in cases of dyspnea.

Early surgical intervention is almost always curative and with a low risk of recurrence, which is greater after recent surgical procedures, young individuals, and incomplete resection [10, 21].

The great difficulty of the management of the left ventricular myxoma is the little experience in the surgical procedure becoming an important challenge. According to Kay et al. [7], the surgical approach can be performed in various ways, among them: through the left atrium and the mitral valve, the ascending aorta, with video assistance, the right atrium and the interatrial septum, through a small longitudinal incision in the left ventricle [7, 22]. As shown in this case report the method used was that of the mitral valve, which presented good results.

\section{Conclusions}

Myxoma is a rare disease of surgical treatment. However, there is still a great difficulty for its management due to the scarcity of cases, as well as the increasing need for publications related to the subject, most of all regarding right and left ventricular myxomas. The current challenge is to make scientific information widely available to health professionals, facilitating clinical and surgical management. The purpose of this publication is to inform and update on this topic that, although not common, is relevant.

\section{References}

[1] Reynen, K. 1996. "Frequency of Primary Tumors of the Heart." Am. J. Cardiol. 77: 107-11.

[2] Manual de Cirurgia Cadiovascular IMIP/INCOR-RHP. 2010. Manual de cirurgia cardiovascular (1st ed.), edited by Moraes, C. R., Moraes, F. Recife: IMIP/INCOR-RHP.

[3] Keshelava, G., Vashakmadze, N., Jaiani, S., Kovziridze, D., and Kurashvili, G. 2018. "Left Ventricular Myxoma with Embolization Causes Acute Infrarenal Aortic Occlusion.” Int. J. Angiol. 27 (1): 43-5

[4] Azad, S., Dutta, N., Roy, C. K., Ramman, T. R., Chandra, N., Radhakrishnan, S., and Iyer, K. S. 2018. "Atypical Left Ventricular Myxoma: Unusual Echocardiographic and Histopathological Features." World J. Pediatr Congenit Heart Surg. Jan 1:2150135117742626.

[5] Wang, Y., Yin, K. H.,, Zhang, Z. Q., Lin, Y., Sun, Y. X., and Wang, C. S. 2017. "Recurrent Left Ventricular Myxoma. Heart, Lung and Circulation.” 27 (6): e82-3.

[6] Simsek, E., Durdu, S., Hodo, B., Yazicioglu, L., and Uysalel, A. 2013. "Left Ventricular Myxoma Producing Cardiac Failure." Heart Surgery Forum, 16 (1): 57-9. https://doi.org/10.1532/HSF98.20121063.

[7] Kay, J. H., Anderson, R. M., Meihaus, J., Lewis, R., Magidson, O., Bernstein, S., and Griffith, G. C. 1959. "Surgical Removal of an Intracavitary Left Ventricular Myxoma." Circulation 20: 881-6.

[8] Hernández-Bringas, O., and Ortiz-Hidalgo, C. 2016. "Histopathological and immunohistochemical features of cardiac myxomas.” Archivos De Cardiología De México 83 (3): 199-208.

[9] Pooja, S., Adriana, L., Vivek, R., and Jagdish, B. 2014. "Molecular Basis of Cardiac Myxomas." Int. J. Mol Sci. 15 (1): 1315-37.

[10] Pérez Díaz, P., Muñoz Ruiz, N., and JuradoRomán, A. 2017. "Left Ventricular Myxoma: Recurrence and Risk of Embolism.” Ann. Thorac Surg. 103 (6): e553. 
[11] Song, X. L., Tang, L., Yang, J., and Li, N. 2013. "Recurrence of Nonfamilial Cardiac Myxoma in the Left Ventricle: A Case Report.” Journal of Clinical Ultrasound Jcu. 42 (9): 576-7.

[12] Kirschner, L. S., Carney, J. A., Pack, S. D., Taymans, S. E., Giatzakis, C., Cho, Y. S., et al. 2000. "Mutations of the Gene Encoding the Protein Kinase A Type I- $\alpha$ Regulatory Subunit in Patients with the Carney Complex." Nature Genetics, 26 (1): 89-92. https://doi.org/10.1038/79238.

[13] Pinede, L., Duhaut, P., and Loire, R. 2001. "Clinical Presentation of Left Atrial Cardiac Myxoma: A Series of 112 Consecutive Cases.” Medicine (Baltimore) 80: 159-72.

[14] Escobar, F., Attié, F., Barrón, J., Marroquín, S., and del Abadiano, J. 2016. "Left Ventricular Myxoma." Archivos De Cardiología De México 74 (4): 290-4.

[15] Tamulevičiūtè, E., Taeshineetanakul, P., Terbrugge, K., et al. 2011. "Myxomatous Aneurysms: A Case Report and Literature Review.” Interv. Neuroradiol 17: 188-94.

[16] Heletz, I., and Abramson, S. V. 2009. "Large Obstructive Cardiac Myxofi-Brosarcoma Is Nearly Invisible on Ransthoracic Echocardiogram." Echocardiography 26: 847-51.
[17] Moreno Martínez, F., Lagomasino Hidalgo, A., Mirabal Rodríguez, R., López Bermúdez, F., and López Bernal, O. 2016. "[Recurrent Left Atrial Myxoma." Archivos De Cardiologia De México 73 (4): 280-3.

[18] Vázquez-Antona, C., Roldán, F., Erdmenger Orellana, J., Romero-Cárdenas, A., Buendía, A., and Vargas-Barrón, J. 2016. "Recurrent Myxoma. Bi and Three-Dimensional Echocardiography Approach." Archivos De Cardiología De México 75 (4): 463-6.

[19] Tarelo-Saucedo, J., Peñaloza-Guadarrama, M., Villela-Caleti, J., García-Cruz, A., Arizmendi-Monroy, D., Martinez-Ramirez, L., et al. 2016. "Surgical Results and Monitoring of Postoperative Atrial Myxomas." Archivos De Cardiología De México 86 (1): 35-40.

[20] Arafat, A A., Abdelwahab, A. A., Sabry, M., and Elsaied, A. 2017. "Left Ventricular Myxoma Associated with Mitral Regurgitation.” J. Card. Surg. 32: 803-4. https://doi.org/10.1111/jocs.13254.

[21] Blondeau, P. 1990. "Primary Cardiac Tumours: French Studies of 533 Cases." Thorac Cardiovasc Surg. 38: 192-5.

[22] Zeitschrift Fur Rheumatologie [Z Rheumatol] 2014 Mar 7. Date of Electronic Publication. 